NAA-SR-7263

PHYSICS

21 PAGES

\title{
REFLECTED REACTOR KINETICS
}

\section{By}

R. W. KEATEN

C. W. GRIFFIN

\section{ATOMICS INTERNATIONAL}

A DIVISION OF NORTH AMERICAN AVIATION, INC. P.O. BOX 309 CANOGA PARK, CALIFORNIA

CONTRACT: AT(11-1)-GEN-8

ISSUED: MARCH 1, 1963 


\section{DISCLAIMER}

This report was prepared as an account of work sponsored by an agency of the United States Government. Neither the United States Government nor any agency Thereof, nor any of their employees, makes any warranty, express or implied, or assumes any legal liability or responsibility for the accuracy, completeness, or usefulness of any information, apparatus, product, or process disclosed, or represents that its use would not infringe privately owned rights. Reference herein to any specific commercial product, process, or service by trade name, trademark, manufacturer, or otherwise does not necessarily constitute or imply its endorsement, recommendation, or favoring by the United States Government or any agency thereof. The views and opinions of authors expressed herein do not necessarily state or reflect those of the United States Government or any agency thereof. 


\section{DISCLAIMER}

Portions of this document may be illegible in electronic image products. Images are produced from the best available original document. 


\section{DISTRIBUTION}

This report has been distributed according to the category "Physics" as given in "Standard Distribution Lists for Unclassified Scientific and Technical Reports" TID-4500 (18th Ed.), August 31, 1962. A total of 675 copies was printed. 


\section{CONTENTS}

Page

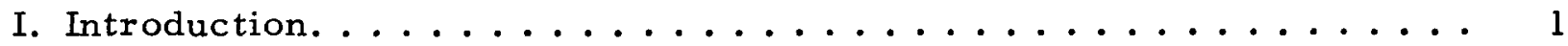

II. Derivation of the Reflected Reactor Transfer Function . . . . . . . 2

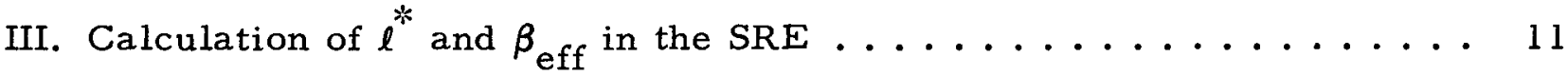

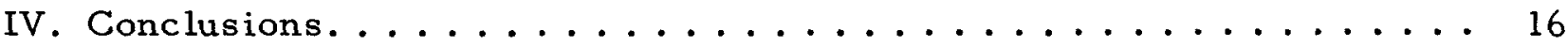

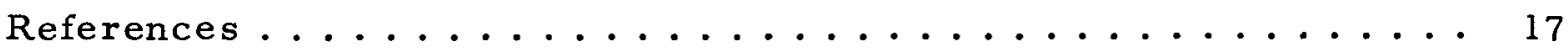

\section{FIGURES}

1. Flow Diagram for Bare Reactor Kinetics . . . . . . . . . . . . 1

2. Flow Diagram for Reflected Reactor Kinetics . . . . . . . . . . . 3

3. Modified Flow Diagram for Reflected Reactor Kinetics . . . . . . . 4

4. Reflected Reactor Transfer Function . . . . . . . . . . . . . . . 10 


\begin{abstract}
The transfer function of a reflected reactor has been derived from a model which is applicable to all reactors. The result is identical to a bare reactor transfer function at low frequencies, provided that the neutron lifetime includes the effect of the reflector. At high frequencies the reflector introduces in effect an additional group of delayed neutrons. A calculation of the effective neutron lifetime in the SRE from the theoretical results was in good agreement with the experimental value.
\end{abstract}




\section{INTRODUCTION}

Kinetic behavior of a bare reactor has been studied extensively, both theoretically and experimentally. These studies have shown that the inhour equation and transfer function derived from the usual form of the kinetics equation give excellent agreement with experimental results.

Reflected reactors have usually been treated by assuming that the same kinetics equations are applicable providing that a modified value of the prompt neutron lifetime is used which includes the effect of the reflector. Measurements of fast transients in the KEWB reactor, however, indicated empirically that treatment of the reflector by an additional delay group (or groups) would give better results. ${ }^{1}$

One theoretical approach to reflected reactor kinetics has been made previously. ${ }^{2}$ The method was applied to a coupled fast-thermal critical assembly to calculate the prompt neutron lifetime. The results agreed well with other determinations. However, attempts to apply this method to the Sodium Reactor Experiment (SRE) give unreasonable values for the lifetime. Further examination showed that this method is inapplicable to reactors with reflectors which make a large contribution to reactivity. This report describes a method of analysis which avoids the above difficulty, and is thus applicable to all reflected reactors. 


\section{DERIVATION OF THE REFLECTED REACTOR TRANSFER FUNCTION}

The general equation governing reactor kinetics is:

$\underset{\text { Rate of Change }}{\text { Neutron Density }}=\begin{gathered}\text { Production } \\ \text { Rate }\end{gathered}-\begin{gathered}\text { Destruction } \\ \text { Rate }\end{gathered}+$ Source

This equation is usually applied to a bare reactor by assuming the "flow diagram" for the neutrons shown in Figure 1. Thus Equation 1 becomes, in the absence of a source,

$$
\frac{d n}{d t}=\frac{(l-\beta) k_{e f f}{ }^{n}}{l^{2 k}}+\sum \lambda_{i} C_{i}-\frac{n}{\ell^{2 k}},
$$

and the associated equations for the delayed neutrons are:

$$
\frac{\mathrm{dC}_{i}}{\mathrm{dt}}=\frac{\beta_{i} \mathrm{k}_{\text {eff }}^{\mathrm{n}}}{\ell^{* k}}-\lambda_{i} \mathrm{C}_{i}
$$

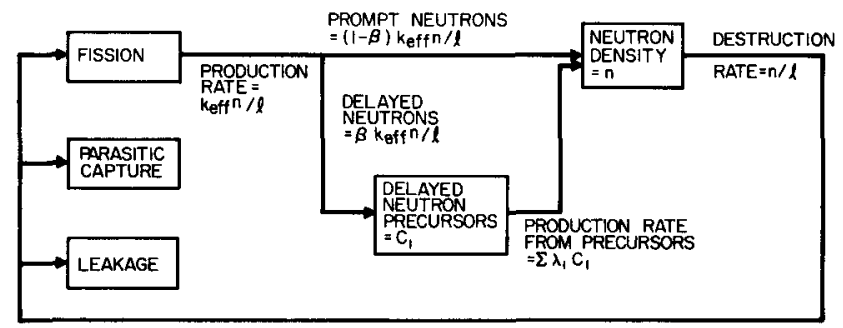

Figure 1. Flow Diagram for Bare Reactor Kinetics

\section{A. PREVIOUS MODEL}

For a reflected reactor, the same approach can be used by treating separately the neutrons in the core and the reflector. However, the neutrons can bounce back and forth from core to reflector many times before being finally absorbed. Thus the diagram shown in Figure 2 applies for a reflected reactor. 


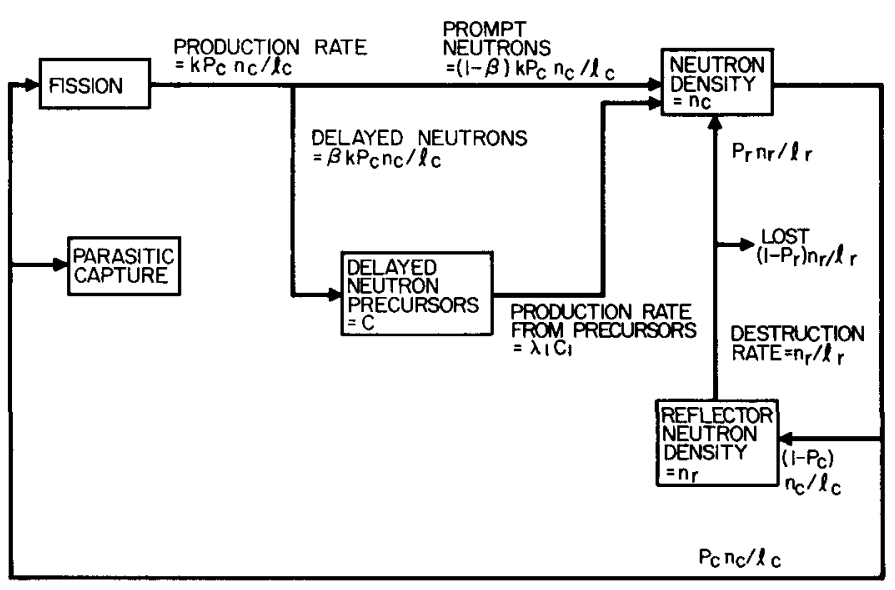

Figure 2. Flow Diagram for Reflected Reactor Kinetics

The definitions of the symbols are:

$$
\begin{aligned}
& \mathrm{n}_{\mathrm{c}}=\text { neutron density in the core } \\
& \mathrm{n}_{r}=\text { neutron density in the reflector } \\
& \mathrm{P}_{\mathrm{c}}=\text { probability that a neutron will remain in the core } \\
& \mathrm{P}_{\mathrm{r}}=\text { probability that a disappearing reflector neutron will reappear in } \\
& \quad \text { the core } \\
& \mathrm{k}=\text { infinite multiplication constant }\left(\mathrm{k}_{\infty}\right) \text { for the core. }
\end{aligned}
$$

The differential equations corresponding to this model are:

$$
\begin{gathered}
\frac{d n_{c}}{d t}=\frac{(1-\beta) P_{c} k n_{c}}{\ell_{c}}+\sum \lambda_{i} C_{i}+\frac{P_{r} n_{r}}{\ell_{r}}-\frac{n_{c}}{\ell_{c}}, \\
\frac{d n_{r}}{d t}=\frac{\left(1-P_{c}\right) n_{c}}{\ell_{c}}-\frac{n_{r}}{\ell_{r}}, \\
\frac{d C_{i}}{d t}=\frac{\beta_{i} k P_{c} n_{c}}{\ell_{c}}-\lambda_{i} C_{i}
\end{gathered}
$$

These equations are identical with those derived by C. E. Cohn. ${ }^{2}$

Although this model is a logical one, it does not give correct results since some neutrons are permitted to go around the "loop" from core to reflector and 
back indefinitely without ever being absorbed. One consequence of this is that the critical equation, obtained by setting all time derivatives in Equation 2 to zero, is

$$
k P_{c}+\left(1-P_{c}\right) P_{r}=1
$$

This is not in agreement with the general form of the critical equation

$$
k \mathfrak{L}=1
$$

where $\mathscr{Q}$, the net nonleakage probability, is some function of $P_{c}$ and $P_{r}$. The discrepancy arises because neutrons returning from the reflector are counted the same as "new" neutrons from fission. In reactors where the reflector makes a large contribution to the reactivity, the error in Equation 2 a would be significant.

\section{B. NEW MODEL}

A model which avoids this difficulty is shown in Figure 3. In this model, neutrons which enter the reflector are not counted in the core until after their

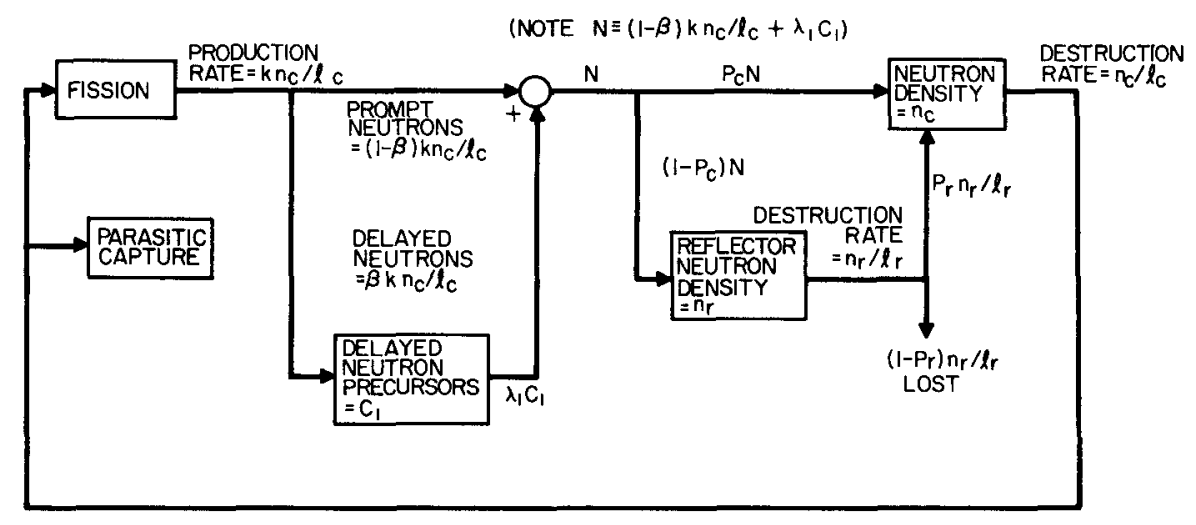

Figure 3. Modified Flow Diagram for Reflected Reactor Kinetics

final exit from the reflector. Although this does not correspond to the physical situation in the reactor, it will give correct results for the kinetic behavior if $\ell_{r}$ and $\ell_{c}$ are defined as the total length of time that neutrons spend in the reflector and core, respectively. In other words the total length of time spent in the two regions is the same in the model as in the reactor, but the chronological order is changed, thus eliminating the "loop." 
The differential equations for this model are:

$$
\begin{gathered}
\frac{d n_{c}}{d t}=\frac{(1-\beta) k P_{c} n_{c}}{\ell_{c}}+\frac{P_{r} n_{r}}{\ell_{r}}+P_{c} \sum \lambda_{i} C_{i}-\frac{{ }_{c}{ }_{c}}{\ell_{c}}, \\
\frac{d n_{r}}{d t}=\frac{(1-B) k\left(1-P_{c}\right) n_{c}}{\ell_{c}}+\left(1-P_{c}\right) \sum \lambda_{i} C_{i}-\frac{n_{r}}{l_{r}}, \\
\frac{d C_{i}}{d t}=\frac{\beta_{i} k_{c}}{\ell_{c}}-\lambda_{i} C_{i}
\end{gathered}
$$

The critical equation, found by setting all time derivatives to zero, is:

$$
\mathrm{k}\left[\mathrm{P}_{\mathrm{c}}+\left(1-\mathrm{P}_{\mathrm{c}}\right) \mathrm{P}_{\mathrm{r}}\right]=1
$$

which now has the same form as the general critical equation.

\section{DERIVATION OF TRANSFER FUNCTION}

To solve equation 3 for the reactor transfer function, the time-varying quantities are replaced by their equilibrium values plus small variations:

$$
\begin{aligned}
& n_{c}=n_{c 0}+\delta n_{c} \\
& n_{r}=n_{r 0}+\delta n_{r} \\
& P_{c}=P_{c 0}+\delta P_{c} \\
& k=k_{0}+\delta k \\
& C_{i}=C_{i 0}+\delta C_{i} .
\end{aligned}
$$

This assumes no variation in $\mathrm{P}_{r}$, which is reasonable for small changes in the core.

The equilibrium values, which are the values at criticality, also satisfy Equation 3. Thus, substituting Equation 5 into Equation 3, subtracting Equation 3 with the critical values inserted, neglecting the products of differentials, and taking Laplace transforms, gives: 


$$
\begin{aligned}
s \delta n_{c} & =\frac{-\delta n_{c}}{\ell_{c}}+\frac{(1-\beta)}{\ell_{c}}\left[P_{c 0} k_{0} \delta n_{c}+n_{0}\left(P_{c 0} \delta k+k_{0} \delta P_{c}\right)\right] \\
& +\frac{P_{r} \delta n_{r}}{l_{r}}+\sum \lambda_{i}\left(C_{i 0} \delta P_{c}+P_{c 0} \delta C_{i}\right), \\
s \delta n_{r} & =\frac{-\delta n_{r}}{\ell_{r}}+\frac{(1-\beta)}{\ell_{c}}\left(1-P_{c 0}\right)\left(n_{c 0} \delta k+k_{0} \delta n_{c}\right)-k_{0} n_{0} \delta P_{c} \\
& +\lambda_{i}\left[\left(1-P_{c 0}\right) \delta C_{i}-C_{i 0} \delta P_{c}\right], \\
s \delta C_{i} & =-\lambda_{i} \delta C_{i}+\frac{\beta_{i}}{\ell_{c}}\left(n_{c 0} \delta k+k_{0} \delta n_{c}\right) .
\end{aligned}
$$

The last one of Equation 6 yields

$$
\delta C_{i}=\frac{\beta_{i}\left(n_{c 0} \delta k+k_{0} \delta n_{c}\right)}{\ell_{c}\left(s+\lambda_{i}\right)}
$$

However, $\mathrm{n}_{\mathrm{c} 0} \delta \mathrm{kcan}$ be neglected since at low frequencies it is much smaller than $\mathrm{k}_{0} \delta \mathrm{n}_{\mathrm{c}}$, and at higher frequencies both terms are negligible. (This same approximation is made in the derivation of the bare reactor transfer function.)

$\mathrm{C}_{\mathrm{i} 0}$ in Equation 6 is the equilibrium solution of the third of Equations 3 where $\mathrm{dC}_{\mathrm{i}} / \mathrm{dt}=0, \mathrm{k}=\mathrm{k}_{0}$ and $\mathrm{n}_{\mathrm{c}}=\mathrm{n}_{\mathrm{c} 0}$; so

$$
\lambda_{i} C_{i 0}=\frac{\beta_{i} k_{0} n_{c 0}}{\ell_{c}}
$$

Now using Equation 8 in the first two equations of 6 , solving the second for $\delta n_{r}$ and substituting the result and Equation 7 into the first of Equation 6 gives: 


$$
\begin{aligned}
\frac{\delta n_{c}}{n_{c 0}}\{s & \left.+\frac{1}{\ell_{c}}-\frac{(1-\beta) k_{0}}{\ell_{c}}\left[P_{c 0}+\frac{\left(1-P_{c 0}\right) P_{r}}{\ell_{r} s+1}\right]-\frac{k_{0}}{\ell_{c}}\left[P_{c 0}+\frac{\left(1-P_{c 0}\right) P_{r}}{\ell_{r} s+1}\right] \sum \frac{\lambda_{i} B_{i}}{s+\lambda_{i}}\right\} \\
& =\frac{(1-\beta)}{\ell_{c}}\left[P_{c 0} \delta k+k_{0} \delta P_{c}\right]+\frac{P_{r}(1-\beta)}{\ell_{c}\left(\ell_{r} s+1\right)}\left[\left(1-P_{c 0}\right) \delta k-k_{0} \delta P_{c}\right] \\
& +\frac{\beta k_{0} \delta P_{c}}{\ell_{c}}\left[1-\frac{P_{r}}{\ell_{r} s+1}\right]
\end{aligned}
$$

where all terms involving $\delta \mathrm{n}_{\mathrm{c}}$ have been taken to the left hand side.

The term

$$
\frac{\beta \mathrm{k}_{0}}{\ell_{\mathrm{c}}}\left[\mathrm{P}_{\mathrm{c} 0}+\frac{\left(1-\mathrm{P}_{\mathrm{c} 0}\right) \mathrm{P}_{\mathrm{r}}}{\ell_{\mathrm{r}} \mathrm{s}+\mathrm{l}}\right]
$$

can be combined with the term including the summation, since $\beta=\sum \beta_{i}$, yielding:

$$
\frac{k_{0}}{\ell_{c}}\left[P_{c 0}+\frac{\left(1-P_{c 0}\right) P_{r}}{\ell_{r} s+1}\right] \sum \frac{\beta_{i} s}{s+\lambda_{i}} .
$$

Now, in this term only, let $\left(\ell_{r} s+1\right)=1$. Although this is valid only at low frequencies, at higher frequencies the entire term is very small, so the error in the transfer function is less than $1 \%$ at any frequency. This approximation is equivalent to neglecting the additional delay of the reflector on the delayed neutrons which enter the reflector.

From Equation 4

$$
k_{o} P_{c 0}+k_{o}\left(1-P_{c 0}\right) P_{r}=1
$$


so solving Equation 9 for $\delta \mathrm{n} / \mathrm{n}_{0}$ gives, letting $\lambda_{\mathrm{r}}=1 / \ell_{\mathrm{r}}$, and $1-\beta=1$ :

$$
\frac{\delta n_{c}}{n_{c 0}}=\frac{P_{c 0} \delta k+k_{0} \delta P_{c}+\frac{\left(1-P_{c 0}\right) P_{r} \delta k-P_{r} k_{0} \delta P_{c}}{\ell_{r}\left(s+\lambda_{r}\right)}}{s \ell_{c}\left[1+\frac{k_{c 0}\left(1-P_{c 0}\right) P_{r}}{\ell_{c}\left(s+\lambda_{r}\right)}+\frac{1}{\ell_{c}} \sum \frac{\beta_{i}}{s+\lambda_{i}}\right]} .
$$

Equation 4 implies that

$$
k_{\text {eff }}=k_{c}+k\left(1-P_{c}\right) P_{r}
$$

so,

$$
\delta \rho=\frac{\delta \mathrm{k}_{\text {eff }}}{\mathrm{k}_{\mathrm{eff}_{0}}}=\mathrm{P}_{\mathrm{c} 0} \delta \mathrm{k}+\mathrm{k}_{0} \delta \mathrm{P}_{\mathrm{c}}+\left(1-\mathrm{P}_{\mathrm{c} 0}\right) \mathrm{P}_{\mathrm{r}} \delta \mathrm{k}-\mathrm{P}_{\mathrm{r}_{0} \mathrm{k}_{0} \delta \mathrm{P}_{\mathrm{c}}}, \ldots
$$

as suming, as before, no change in $\mathrm{P}_{\mathrm{r}}$.

At low frequencies the numerator in Equation 10 is therefore $\delta \rho$. At higher frequencies the numerator is a dynamic reactivity, in which a change in the probability of neutrons escaping to the reflector is affected by the frequency of the change. This is physically reasonable, since a very fast change will have less effect due to the delay associated with the reflector.

Thus the transfer function of a reflected reactor is:

$$
G_{r}(s)=\frac{\delta n_{c} / n_{c 0}}{\delta \rho / \beta}=\frac{1}{\frac{s \ell_{c}}{\beta}\left[1+\frac{k_{c 0}\left(1-P_{c 0}\right) P_{r}}{\ell_{c}\left(s+\lambda_{r}\right)}+\frac{\beta}{\ell_{c}} \sum_{i} \frac{\beta_{i} / \beta}{s+\lambda_{i}}\right]} .
$$

\section{DISCUSSION}

At low frequencies where $\omega \ll 1 / \ell_{r}$, the transfer function is identical to the bare reactor transfer function: 


$$
G(s)=\frac{1}{\frac{s \ell^{*}}{\beta}\left[1+\frac{\beta}{\ell^{* *}} \sum_{i} \frac{\beta_{i} / \beta}{s+\lambda_{i}}\right]},
$$

providing that we define

$$
\ell^{*}=\ell_{\mathrm{c}}+\mathrm{k}_{\mathrm{c} 0}\left(1-\mathrm{P}_{\mathrm{c} 0}\right) \mathrm{P}_{\mathrm{r}} \ell_{\mathrm{r}}
$$

Thus the effective lifetime is the core lifetime plus the reflector lifetime weighted by the fraction of neutrons which enter and return from the reflector.

At higher frequencies the reflector in effect introduces a seventh group of delayed neutrons:

$$
G_{r}(s)=\frac{1}{\frac{\ell_{c} s}{\beta}\left[1+\frac{\beta}{\ell_{c}} \sum_{i=1}^{i=7} \frac{\beta_{i} / \beta}{s+\lambda_{i}}\right]},
$$

where

$$
\begin{gathered}
\lambda_{7} \equiv \lambda_{r}=\frac{1}{l_{r}}, \\
\beta_{7} \equiv k_{c 0}\left(1-P_{c 0}\right) P_{r} .
\end{gathered}
$$

Note that in Equation 15,

$$
\beta \equiv \sum_{i=1}^{i=6} \beta_{i},
$$

i. e., it does not include the neutrons from the reflector. This is physically reasonable because the delay associated with the reflector is very much shorter than that of the real delay groups. Thus the reactivity required for prompt criticality is not radically changed by the presence of a reflector. 
Figure 4 shows a plot of the transfer function of a typical graphite-moderated and -reflected thermal reactor, in which $\ell_{C}$ and $\ell_{r}$ are the same order of magnitude. In this case, the only perturbation of the bare reactor transfer function is well above the region usually considered for lifetime measurements. However, if the reflector lifetime is much longer than the core lifetime, then the transfer function may be distorted in the region of the $\ell^{*} / \beta$ break-off, and the bare reactor transfer function would not represent an adequate approximation.

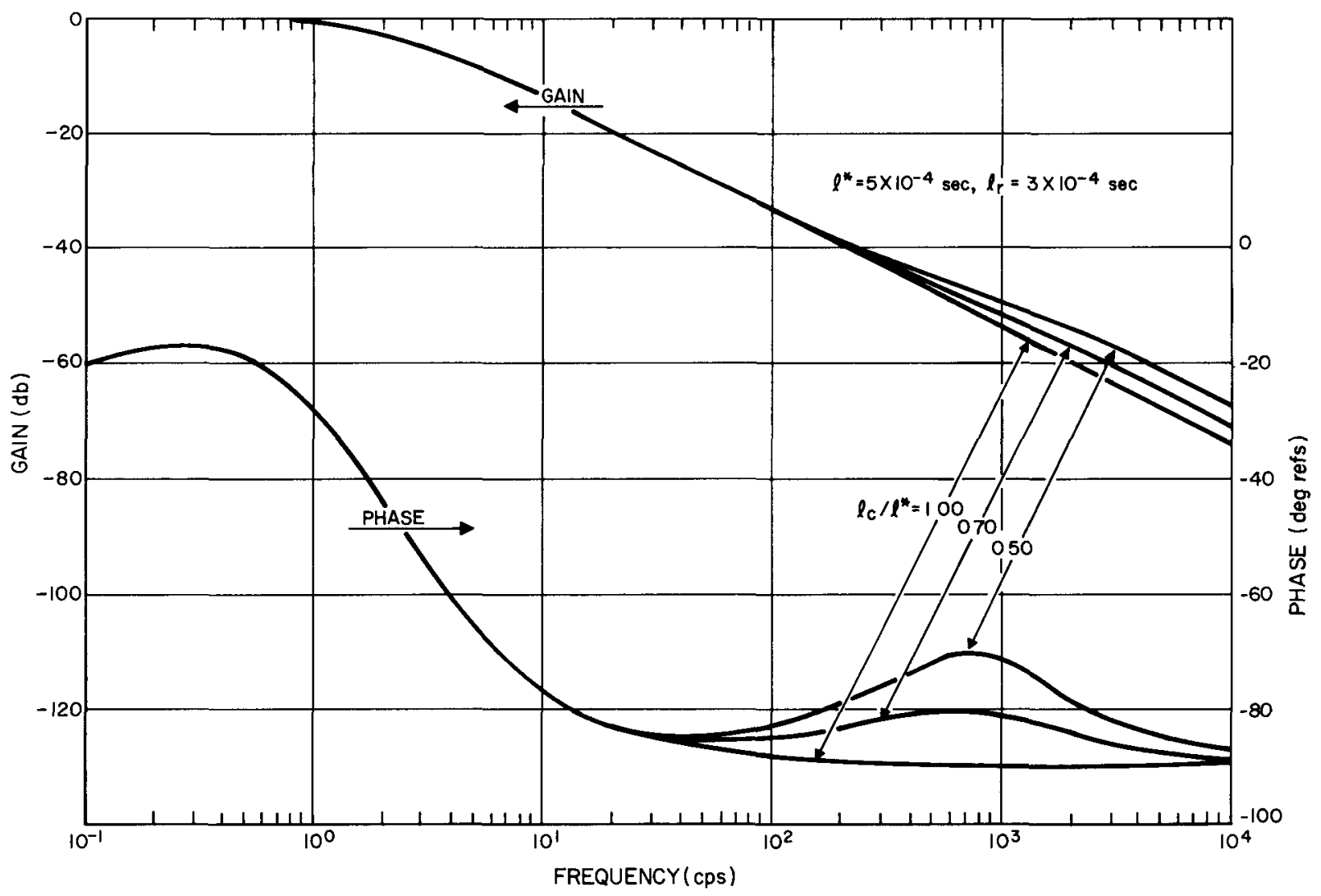

Figure 4. Reflected Reactor Transfer Function

The transfer function derived above is identical to that of Cohn ${ }^{2}$ except for the expression for effective lifetime, as discussed in the following section. 


\section{CALCULATION OF $\ell^{*}$ AND $\beta_{\text {eff }}$ IN THE SRE}

A calculation of $\ell^{* *}$ and $\beta_{\text {eff }}$ for the second core loading of the SRE was used for comparison with the results of the transfer function measurement. The method was a combination of the results from the preceeding derivation with a two-group analysis of the kinetics of a bare reactor, ${ }^{3}$ which yields the following expressions:

$$
\begin{gathered}
\beta_{\text {eff }}=\beta\left(k_{d}\right), \\
\ell_{c}=\ell_{c t h}\left(\frac{1-\nu \Sigma_{1}}{\Sigma l_{r}}\right)+\ell_{c f},
\end{gathered}
$$

where

$$
\begin{aligned}
k_{d}= & \text { effective multiplication constant for delayed neutrons } \\
\ell_{c f}, \ell_{c t h}= & \text { prompt neutron lifetime in the core for fast and thermal groups, } \\
& \text { respectively } \\
\ell_{c}= & \text { total mean prompt neutron lifetime in the core } \\
\Sigma_{l_{f}} & \text { fission cross section in fast group } \\
\Sigma_{l_{r}}= & \text { total removal cross section from fast group } \\
\nu & =\text { number of fission neutrons per fission. }
\end{aligned}
$$

Equation 16 can be applied directly to the SRE, since the reflector affects $\beta_{\text {eff }}$ only in the value of $k_{d}$. Since $k_{d}$ is identical to $k_{\text {eff }}$ except for the fast nonleakage probability, then for a critical reactor where $\mathrm{k}_{\text {eff }}=\mathrm{l}$ :

$$
\mathrm{k}_{\mathrm{d}}=\mathrm{e}^{\mathrm{B}^{2}\left(\tau_{\mathrm{p}}-\tau_{\mathrm{d}}\right)}
$$

where $\tau_{p}$ and $\tau_{d}$ are the fission to thermal ages for prompt and delayed neutrons, respectively, and $\mathrm{B}^{2}$ is the effective buckling for a reflected reactor. The values of $\tau$ were obtained by the usual method, 4 and $\mathrm{B}^{2}$ was determined from a two-group 
calculation on the reflected reactor by requiring that $e^{-B^{2}} \tau_{\text {eff }}[$ where $\tau$ eff $\left.=(1-\beta) \tau_{p}+\beta \tau_{d}\right]$ give the correct fast nonleakage probability. Using the se values, $k_{d}=1.058$ and $\beta_{\text {eff }}=0.0069$.

The calculation of $\ell^{* *}$ used the results of the derivation of the reflected trans fer function:

$$
\ell^{*}=\ell_{\mathrm{c}}+\mathrm{k}_{\mathrm{c} 0}\left(1-\mathrm{P}_{\mathrm{c} 0}\right) \mathrm{P}_{\mathrm{r}} \ell_{\mathrm{r}}
$$

The value of $\ell_{c}$ is obtained from Equation 17 with: ${ }^{5}$

$$
\begin{aligned}
& \ell_{\mathrm{cth}}=\frac{1}{\mathrm{v}_{\mathrm{th}}\left(\sum_{\mathrm{a}_{\mathrm{th}}}+\mathrm{D}_{\mathrm{th}} \mathrm{B}_{\mathrm{c}}^{2}\right)} \text {, } \\
& \ell_{\mathrm{fth}}=\frac{\lambda_{\mathrm{s}} \sqrt{2 \mathrm{~m}}}{\xi}\left[\frac{1}{\sqrt{E_{\mathrm{th}}}}-\frac{1}{\sqrt{E_{\text {fission }}}}\right] \text {, }
\end{aligned}
$$

where:

$$
\begin{aligned}
& \mathrm{V}_{\mathrm{th}}=\text { average velocity of thermal neutrons } \\
& \Sigma_{a_{\text {th }}}, D_{\text {th }}=\text { thermal absorption cross section and diffusion coefficient, } \\
& \text { respectively, in the core } \\
& \lambda_{\mathrm{S}}=\text { mean free path of fast neutrons } \\
& \mathrm{m}=\text { mass of neutron }=1.66 \times 10^{-24} \mathrm{gm} \\
& \xi=\text { logarithmic decrement of energy lost per collision } \\
& E_{\text {th }}, E_{\text {fission }}=\text { ene rgy of thermal and fission neutrons, respectively } \\
& \mathrm{B}_{\mathrm{C}}^{2}=\text { geometric buckling of bare core: } \\
& \mathrm{B}_{\mathrm{c}}^{2}=\left(\frac{2.405}{\mathrm{R}_{\text {core }}}\right)^{2}+\left(\frac{\pi}{\mathrm{H}_{\text {core }}}\right)^{2} .
\end{aligned}
$$


Using previously reported values for the SRE, ${ }^{6} \ell_{\text {cth }}=5.1 \times 10^{-4} \mathrm{sec}$ and $\ell_{\text {fth }}$ $=0.5 \times 10^{-4} \mathrm{sec}$.

The term $\Sigma_{1_{f}} / \Sigma_{l_{r}}$ is the fraction of neutrons which causes fissions before becoming thermal. This value is very difficult to calculate accurately in the SRE. A rough estimate was made using the infinite dilution resonance integral for $\mathrm{U}^{235}$ as the effective cross section in the fuel, and then computing a fuel resonance disadvantage factor by the same method as is used for thermal flux calculations. ${ }^{6}$ The result was $\Sigma_{l_{f}} / \Sigma_{1_{r}}=0.06$. Using the above values in Equation 17:

$$
\ell_{c}=4.8 \times 10^{-4} \mathrm{sec} .
$$

The thermal neutron lifetime in the reflector was obtained by a Monte Carlo calculation which followed 200 neutrons injected into the reflector. This calculation showed that the average number of mean free paths traveled before returning to the core is 17 , and the probability of return is 0.89 . A similar calculation showed that the neutrons leaving the reflector have a probability of 0.60 of returning again to the reflector before being absorbed in the core.

Let $f_{r}$ be the probability that a reflector neutron will return to the core, and let $f_{c}$ be the probability that a neutron leaving the reflector will return to the reflector before being absorbed in the core. Then the probability of making exactly one pass through the reflector and returning to the core is $f_{r}\left(1-f_{c}\right)$. For two passes, the probability is $f_{r} f_{c} f_{r}\left(1-f_{c}\right)$, etc. Thus the total mean distance traveled in the reflector before finally being absorbed in the core is:

$$
\begin{aligned}
D_{r} & =\frac{f_{r}\left(1-f_{c}\right) d_{r}+\left(f_{r} f_{c}\right) f_{r}\left(1-f_{c}\right) 2 d_{r}+\left(f_{r} f_{c}\right)\left(f_{r} f_{c}\right) f_{r}\left(l-f_{c}\right) 3 d_{r}+\ldots}{f_{r}\left(1-f_{c}\right)+f_{r} f_{c} f_{r}\left(1-f_{c}\right)+f_{r} f_{c} f_{r} f_{c} f_{r}\left(1-f_{c}\right)+\ldots} \\
& =\frac{d_{r}}{1-f_{r} f_{c}}
\end{aligned}
$$

where

$$
\begin{aligned}
\mathrm{d}_{\mathbf{r}}= & \text { mean distance traveled during one pass through the reflector } \\
\mathrm{D}_{\mathbf{r}}= & \text { mean total distance traveled in the reflector before the final return } \\
& \text { to the core. }
\end{aligned}
$$


Thus the lifetime of thermal neutrons in the reflector is

$$
\ell_{r_{\text {th }}}=\frac{D_{r}}{V_{\text {th }}}=\frac{d_{r}}{V_{\text {th }}\left(1-f_{r_{c}}\right)},
$$

where $\mathrm{V}_{\text {th }}$ is the mean velocity of thermal neutrons in the reflector.

Neutrons which spend time in the reflector only as fast neutrons have the same lifetime as those which remain in the core, since the slowing-down times in the core and reflector are the same. For computational ease this effect is taken into account by as suming that the slowing-down time in the reflector is zero, and that a neutron which slows down in the reflector has the same lifetime in the core as those which slow down in the core.

The Monte Carlo calculation indicated that about 25\% of the fast neutrons which enter the reflector never appear there as a thermal neutron, so for these neutrons $l_{r}=0$. The remaining $75 \%$ of those which enter as fast neutrons also appear there as thermal neutrons, and have the same mean lifetime in the reflector as those which first enter as thermal neutrons. The probability that a neutron will remain in the core is:

$$
P_{c 0}=\frac{e^{-B_{c}^{2} \tau}}{1+L^{2} B_{c}^{2}} \text {, }
$$

and the probability of entering the reflector as a fast neutron is:

$$
P_{f}=1-e^{-B_{c}^{2} \tau}
$$

so the probability of first entering as a thermal neutron is:

$$
P_{t h}=\left(1-P_{c 0}\right)-P_{f}=\frac{\left(L^{2} B_{C}^{2}\right) e^{-B_{C}^{2} \tau}}{1+L^{2} B_{C}^{2}} \text {. }
$$


Therefore, the mean 'lifetime' in the reflector is obtained by weighting the respective lifetimes by the fraction of neutrons:

$$
\ell_{r}=\frac{\left(1-e^{\left.-B_{C}^{2} \tau\right)}\right.}{1-\frac{e^{-B_{C}^{2} \tau}}{1+L^{2} B_{C}^{2}}}\left[0.25(0)+0.75\left(\ell_{r_{t h}}\right)\right]+\frac{\frac{\left(L^{2} B_{c}^{2}\right) e^{-B_{C}^{2} \tau}}{1+L^{2} B_{C}^{2}}}{1-\frac{e^{-B_{C}^{2} \tau}}{1+L^{2} B_{c}^{2}}}=2.9(10)^{-4} \mathrm{sec}
$$

Using this and the calculated lifetime in the core in Equation 14:

$$
\ell^{* *}=5.5(10)^{-4} \mathrm{sec}
$$

This agrees very well with the experimental value ${ }^{7}$ of $5.2(10)^{-4} \mathrm{sec}$ Cohn's $^{2}$ expression for the effective lifetime is (in the present notation):

$$
\ell^{* k}=\frac{\ell_{c}+k_{c 0}\left(1-P_{c 0}\right) P_{r_{r}}{ }_{r}}{k_{c 0} P_{c 0}},
$$

yielding a value of $\ell^{*}=7.2(10)^{-4} \mathrm{sec}$, which is not in agreement with the experimental value. Thus, the present derivation yields a bette $r$ value for the effective neutron lifetime. 


\section{CONCLUSIONS}

a) Bare reactor kinetics provides an accurate method of treating reflected reactors at frequencies more than one decade below the break frequency of the reflector, which is about $1000 \mathrm{cps}$ for graphite reflectors.

b) At higher frequency the reflector introduces, in effect, an additional group of delayed neutrons.

c) The reactivity corresponding to prompt criticality is not affected by the reflector.

d) The method of calculating prompt neutron lifetime discussed in this report yields results in good agreement with experiment. 


\section{REFERENCES}

1. J. W. Flora et al., "Kinetic Experiments on Water Boilers "A" Core Report - Part 1: Program History, Facility Description and Experimental Results," NAA-SR-5415, March 1962.

2. C. E. Cohn, "Reflected Reactor Kinetics," Trans. Am. Nuclear Soc., Vol. 4, No. 1, June 1961 .

3. E. C. Toops, "Reactor Kinetics," Trans. Am. Nuclear Soc., Vol. 4, No. 1, June 1961 .

4. F. L. Fillmore, "Two-Group Calculations of the Critical Core Size of the SRE Reactor," NAA-SR-1517 (Rev.), January 1959.

5. S. Glasstone and M. C. Edlund, The Elements of Nuclear Reactor Theory," D. Van Nostrand, Inc. (1952).

6. R. W. Keaten, "Reactivity Worth of Sodium in Sodium Cooled Reactors," NAA-SR -5904, June 1961.

7. C. W. Griffin and R. W. Keaten, "Oscillation Measurements in the SRE," NAA-SR-7264 (to be published). 